\title{
The global non-communicable diseases epidemic and association between dietary factors and non-communicable diseases by income levels
}

\section{Abstract}

Non-communicable diseases (NCDs) share common risk factors as poor dietary intakes, especially among low-income populations worldwide. However, the diet-related health burden by country income levels remains unclear. We assessed the current prevalence of NCDs and the association between selected dietary factors and NCDs by income levels. Data were obtained from the World Health Organization, Food and Agriculture Organization, and World Bank, and 151 countries were included in the analysis weighted by the total population size. Linear regression was used to find the association between metabolic risk factors and health-related behaviors by income levels. The prevalence of raised fasting blood glucose and total cholesterol, overweight, and obesity were lowest in lower and middle income countries, but prevalence of raised blood pressure and NCD deaths under age 70 were highest in lower and middle income countries ( $\mathrm{p}$ for trend $<0.001$ ). The proportion of carbohydrates and alcohol consumer were highest, and vegetable, milk supply, insufficient activity were lowest in lower and middle income countries. In high income countries, raised fasting blood glucose level were negatively associated with vegetable consumption $(\beta=-0.05 \mathrm{CI}[-0.08,-0.02])$, and alcohol consumption, fat intake $(7.94[1.82,14.06])$, and sugar and sweetener supply $(0.04[0.01,0.07])$ were associated with overweight. In low income countries, overweight was associated with vegetable oil $(0.03[0.00,0.05])$. In this study, different relationship between diets and NCDs trends were found across country income levels. Appropriate health policies for each group of countries by income are needed to solve the increasing challenges of NCDs.

This work was carried out with the support of "Cooperative Research Program for Agriculture Science and Technology Development (Project No. PJ PJ01317001)” Rural Development Administration, Republic of Korea.

\section{Conflict of Interest}

This work was carried out with the support of "Cooperative Research Program for Agriculture Science and Technology Development (Project No. PJ PJ01317001)” Rural Development Administration, Republic of Korea. 